\title{
Total body irradiation using VMAT (RapidArc): A planning study of a novel treatment delivery method
}

\author{
Santam Chakraborty ${ }^{1}$, Suja Cheruliyil ${ }^{2}$, Reshmi K Bharthan ${ }^{2}$, Geetha Muttath ${ }^{1}$ \\ ${ }^{I}$ Department of Radiation Oncology, Malabar Cancer Centre, Thalassery, Kerala, India \\ ${ }^{2}$ Division of Radiation Physics, Department of Radiation Oncology, Malabar Cancer Centre, Thalassery, Kerala, India
}

Received December 24, 2014; Revised February 04, 2015; Accepted February 10, 2015; Published Online February 26, 2015

\section{Original Article}

\begin{abstract}
Purpose: To evaluate the feasibility of using volumetric modulated arc therapy (VMAT) using RapidArc to deliver total body irradiation (TBI) treatment. Methods: VMAT planning was performed a whole body computed tomography (CT) data set using Rapid Arc. The planning target volumes included entire body trimmed to $3 \mathrm{~mm}$ below the skin. The organs at risk included the lungs and kidneys. A dose of $12 \mathrm{~Gy}$ in 10 fractions was prescribed to the target volume. The VMAT-TBI technique consisted of three isocentres and three overlapping arcs: the head and neck, the chest, and the pelvis. The plans were prescribed to ensure, at a minimum, 95\% planning target volume dose coverage with the prescription dose (percentage of volume receiving dose of $12 \mathrm{~Gy}$ was $95 \%$ ) and maximum dose of $109.8 \%$. Mean dose to lung was restricted at 8.6 Gy. Results: The total body volume in the study was $15469 \mathrm{~cm}^{3}$ and the PTV volume was $11322 \mathrm{~cm}^{3}$. The mean dose to PTV was $104 \%$. The homogeneity index was 0.09 . Sparing of normal tissues with adequate coverage of skeletal bones was shown to be feasible with Rapid Arc. The study demonstrates that VMAT is feasible for TBI treatment. Unlike conventional TBI chest wall boost with electrons was not required. Conclusion: The technique for total body irradiation using RapidArc VMAT was found feasible and is undergoing further studies prior to clinical use.
\end{abstract}

Keywords: Total Body Radiation; Volumetric Modulated Arc Therapy; RapidArc; Multiple Isocenters

\section{Introduction}

Total body irradiation (TBI) with mega-voltage photon beams has been accepted as an important component of management for a number of hematologic malignancies, generally as part of bone marrow conditioning regimens. The goal of the treatment is to ensure that the prescription dose is delivered with dose homogeneity of $\pm 10 \%$ at the patient mid-line. ${ }^{1}$

Given the large volume of radiation involved it is not surprising that several techniques have been developed to deliver TBI. The most commonly recommended technique utilizes parallel opposed antero-posterior (AP-PA) beams to ensure a homogenous and uniform dose. This beam arrangement usually requires the patient to be treated in a standing or sitting position at extended SSD. ${ }^{2}$ This is usually coupled with partial transmission blocks to shield the lungs. This is usually followed by an electron boost to shielded areas to ensure adequate doses. In order to get acceptable dose variation across the mid line $10 \mathrm{MV}$ or higher photon energies are required $( \pm 30 \%$ if skin dose is included).

However the disadvantage with this technique is that it requires a sick patient to stand for prolonged periods of time often in excess of 40 minutes. ${ }^{3}$ In addition the reproducibility of treatment position is less and positioning of the shields is cumbersome. Hence several new techniques have been designed, some even incorporating innovative machines to deliver TBI in a more simple way. Some of these techniques include treatment using lateral opposing fields, ${ }^{2}$ step translation and dynamic field matching ${ }^{4}$ and recently helical tomotherapy. ${ }^{5}$ While these techniques are innovative they are also limited in their applicability to the institute or innovator of origin. Further in institutes with limited machine availability as in India these techniques are difficult to apply. Time on the machine is a precious resource and treatment time in excess of 40 minutes for a single patient is difficult to justify especially in our setting.

In recent years, TBI has been planned using Helical Tomotherapy. Dosimetrically the dose distribution is more homogeneous as compared to standard extended SSD AP-PA treatment. ${ }^{6}$ It has the advantage that the patient can be treated in supine position without requiring compensators, specialized lung shields and beam spoilers. However, the treatment time of more than one hour with this technique

Corresponding author: Santam Chakraborty; Department of Radiation Oncology, Malabar Cancer Centre, Thalassery, Kerala, India.

Cite this article as: Chakraborty S, Cheruliyil S, Bharthan R, Muttath G. Total body irradiation using VMAT (RapidArc): A planning study of a novel treatment delivery method. Int J Cancer Ther Oncol 2015; 3(2):03028. DOI: 10.14319/ijcto.0302.8

[A part of this research was presented at Association of Medical Physicists of India -2013, Annual Meeting, which was held from November 13-16, 2013 in Kolkata, India] 
may limit its clinical application. Volumetric modulated arc therapy (VMAT) is able to increase the treatment delivery efficiency while increasing the target conformality and reducing the radiation dose to organs at risk (OARs). During a VMAT treatment, the gantry rotates around the patient while the beam is modulated continuously. Both the dose rate and gantry speed can be modulated to achieve optimal dose distribution. Studies showed that VMAT could achieve satisfactory dose conformity and normal organ sparing for a variety of treatment sites. ${ }^{7}$

Use of VMAT to deliver total marrow irradiation (TMI) has also been reported by several authors. ${ }^{8,9}$ Surucu et al. have shown that that median dose difference between TLD measured and TPS measured dose was $0.5 \%{ }^{10}$ Han et al. compared VMAT and Helical Tomotherapy and showed that use of VMAT TMI could achieve comparable target volume coverage, organ at risk doses with reduced beam on time. ${ }^{11}$ However given the difference in the difference of target volume between TMI and TBI applicability of VMAT for delivering TBI remains to be investigated. ${ }^{12}$

The purpose of this study is to develop a total body irradiation technique that does not require additional devices or sophisticated processes. The technique aims to deliver a uniform dose to the entire body while keeping the lung dose within the tolerance level using VMAT.

\section{Methods and Materials}

Pre-existing computed tomography (CT) data-set of an adult patient, treated previously with craniospinal radiation, imaged from the vertex of the skull up to the mid-thigh was used for the planning purpose. The planning target volumes included entire body trimmed to $3 \mathrm{~mm}$ below the skin. The organs at risk included the lungs and kidneys.

Treatment planning was performed on the Eclipse 10.0 platform (Varian Medical Systems, Palo Alto, California, USA). A dose of $12 \mathrm{~Gy}$ in 10 fractions was prescribed to the target volume. Dose was normalized so that $95 \%$ of the prescribed dose covered $95 \%$ of the PTV. Planning objectives for PTV aimed to limit the minimum and maximum doses. 6 MV photon beam was used for the arc fields. A 120-leaf multi-leaf collimator (MLC) was used for beam modulation. There were 60 leaves on each side of the MLC, with each of the central 40 leaves projecting a $5 \mathrm{~mm}$ width at $100 \mathrm{~cm}$ source-to-surface distance (SSD), and each of the peripheral 20 leaves projecting a $10 \mathrm{~mm}$ width at $100 \mathrm{~cm}$ SSD.

The maximum dose rate used was 200 monitor units (MU) per minute. The maximum gantry rotation speed was 4.8 degree/s. Due to physical limits to the maximum MLC leaf extensions as well as the maximum jaw size; multiple fields were needed to deliver treatment to the complete target volumes. Six arc fields, arranged along the patient's longitudinal axis, were used. The range of gantry rotation angles was from 0-179 for all the arc fields. For efficient treatment delivery, each arc field always had the opposite gantry rotation direction in relation to adjacent fields, so that after each arc field rotation, the gantry will be at the starting position of the next field. The six arcs had a total number of 3 isocentres using asymmetric jaw settings to cover the entire PTV length. Field width was set to $40 \mathrm{~cm}$, while field length was $15 \mathrm{~cm}$.

For each arc field in the VMAT plans, 177 control points were defined, each corresponding to an arc segment of about 2 degree. Progressive resolution algorithm was used for arc field optimization, and the anisotropic analytical algorithm (AAA) was used for final dose calculation, with a calculation grid size of $0.25 \mathrm{~cm}$. Plan was evaluated as per the institutional recommendations.

The median dose (D50) and the dose covering 2\% of the volume (D2) were evaluated for normal organs and target volumes. The percentage target volume that received at least 12 Gy (V12) was used for the evaluation of target dose coverage. Plan is quantitatively evaluated from dose-volume-histogram (DVH) analysis, assessing for PTV: mean dose, percentage of volume receiving $110 \%$ of the prescribed dose and target homogeneity index (HI) defined as D2\%-D98\%: for OARs, the analysis included the median dose (D50\%) and D2\%.

Plan quality assurance was performed using an Octavius II phantom with a PTW 729 ionization chamber (Physikalish-TechnischeWerkstätten-Freiburg, Freiburg, Germany) array. The verification plan consisted of all 3 plans delivered to a single isocenter. Gamma evaluation was done using Low's method with a dose difference of $3 \%$ and distance to agreement of $3 \mathrm{~mm} .{ }^{13}$ Results were analyzed on the VeriSoft software version 4.2 (Physikalish- TechnischeWerkstätten-Freiburg, Freiburg, Germany).

\section{Results}

The total PTV volume was 11322 cc while the volume of lung and kidneys were $664 \mathrm{cc}$ and $144.1 \mathrm{cc}$, respectively. For this initial planning study no further organs at risk were defined. The total length of the target volume was $63 \mathrm{~cm}$. An optimization PTV was created by trimming the PTV by $3 \mathrm{~mm}$ below the skin surface in order to ensure better plan optimization as per our previous clinical experience in other sites. Further in order to ensure adequate sparing of the kidney and lung dummy structures were created by expanding the contours of these organs by $3 \mathrm{~mm}$. The optimization PTV was trimmed from the lung, kidney and this dummy structure. Couch structures were used in addition to ensure that beam attenuation through the couch was accounted for. 
Table 1 lists the optimization objectives used for the planning purpose. Normal tissue optimization (NTO) parameter was not used. Optimization was continued for four levels till desired optimum solution was obtained. The total time taken for the initial field placement and optimization was 40 minutes. After the initial optimization the plans were calculated and normalized to ensure minimum target coverage as per the criteria mentioned above.

TABLE 1: Planning objectives used in VMAT TBI.

\begin{tabular}{llllll}
\hline \hline Type & Volume & Limit & Vol[\%] & Dose(cGy) & Priority \\
\hline Point & Kidney & Upper & 0 & 1200 & 30 \\
Point & Kidney & Lower & 100 & 900 & 50 \\
Mean & Kidney & NA & NA & 1000 & 40 \\
Point & Lung & Upper & 0 & 1200 & 30 \\
Point & Lung & Lower & 100 & 800 & 50 \\
Mean & Lung & NA & NA & 850 & 50 \\
Point & PTV Body & Upper & 0 & 1220 & 65 \\
Point & PTV Body & Lower & 100 & 1200 & 65 \\
\hline \hline
\end{tabular}

NA = Not applicable

Portions of the optimization PTV receiving more than 107\% and less than $95 \%$ was delineated. Subsequently further optimization was performed using the original plan as the base plan. Higher priorities were assigned to these newly created dummy structures to eliminate overdose and increase dose to volumes receiving overdose. This process was repeated 4 times till an optimum plan was generated which met our predefined acceptability criteria. The dosimetric results obtained in this plan for the target volumes and various organs at risk are presented in Table 2 and 3, respectively.

TABLE 2: Target volume dose. PTV $\mathrm{D}_{98}$ and $\mathrm{D}_{2}$ are minimum dose to $98 \%$ and $2 \%$ volumes, respectively. PTV D 50 is the median dose.

\begin{tabular}{ll}
\hline \hline PTV D98 & $1132.68 \mathrm{cGy}$ \\
PTV D2 $_{2}$ & $1251.48 \mathrm{cGy}$ \\
PTVD50 & $1202 \mathrm{cGy}$ \\
PTV Mean dose & $1200 \mathrm{cGy}$ \\
PTV 3D Dose Maximum & $1317.8 \mathrm{cGy}$ \\
PTV 3D Dose Minimum & $903.1 \mathrm{cGy}$ \\
\hline \hline
\end{tabular}

TABLE 3: Organ at risk doses. The $\mathrm{D}_{98}, \mathrm{D}_{67}, \mathrm{D}_{33}$ and $\mathrm{D}_{2}$ are minimum dose to $98 \%, 67 \%, 33 \%$ and $2 \%$ volumes, respectively. V8 is the percentage volume receiving $8 \mathrm{~Gy}$.

\begin{tabular}{lllllll}
\hline OAR & Dmean & $\mathrm{D}_{98}$ & $\mathrm{D}_{2}$ & $\mathrm{D}_{67}$ & $\mathrm{D}_{33}$ & V8 \\
\hline Lung & 864.9 & 761.27 & 1111 & 798.5 & 690.462 & $65 \%$ \\
Kidney & 985.7 & 869.48 & 1141 & 945 & 1021 & NA \\
\hline \hline
\end{tabular}

Color wash dose displays (Figure 1, 2 and 3) show relative sparing of the organs at risk with homogeneous coverage of the rest of the body. Dose volume histograms are presented in Figure 4 .

The total time taken for optimization and calculation of the plans was 5 hours. Plan quality assurance revealed that percentage of pass pixels was $93 \%$ using the Lows criteria at \pm $3 \%$ dose difference and a distance to agreement of $3 \mathrm{~mm}$ for the composite plan.

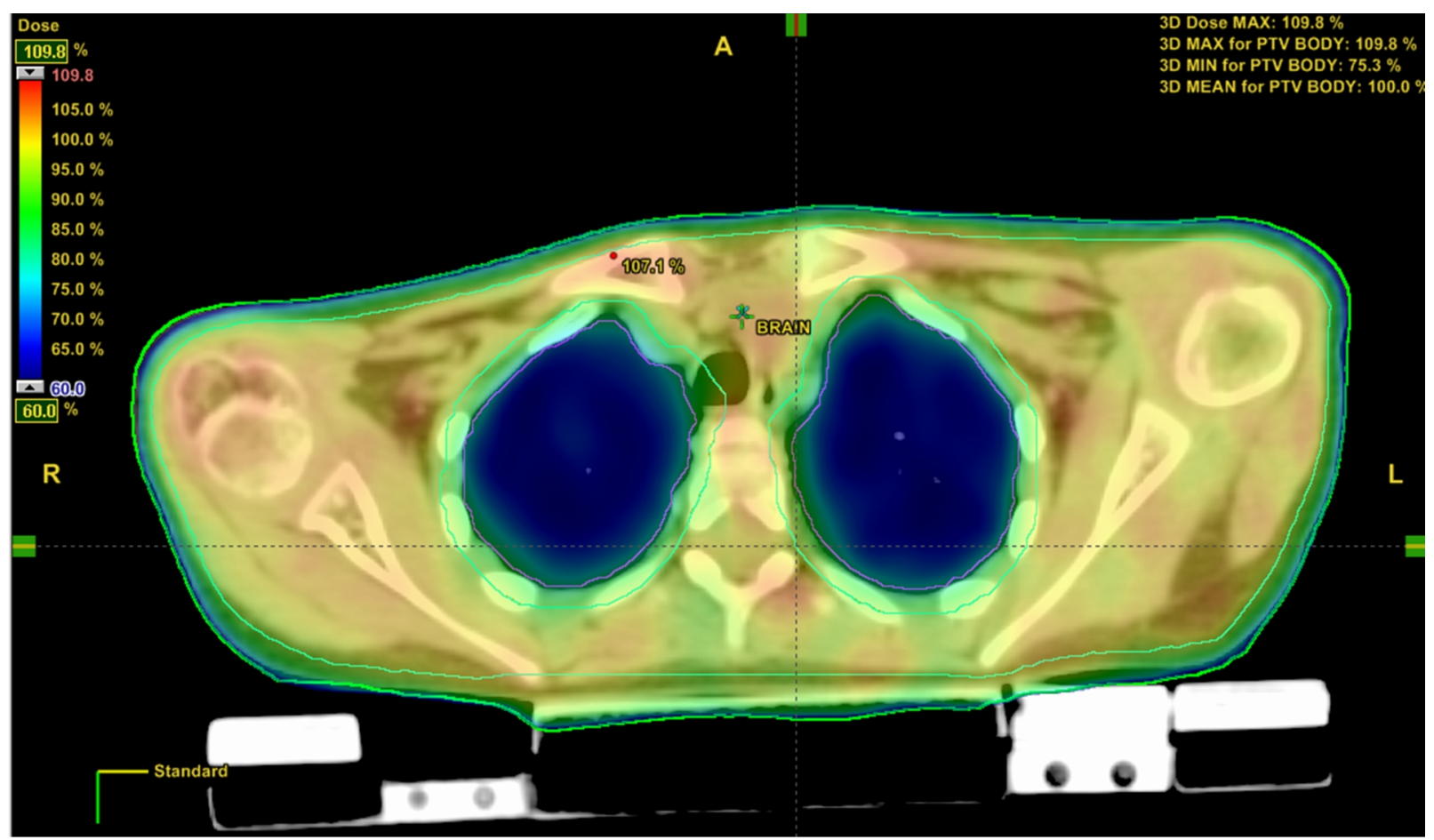

FIG. 1: RapidArc volumetric arc therapy TBI isodose distributions in color wash - Thoracic axial. Isodose lines shown correspond to: 7.2 Gy $(60 \%)$. 


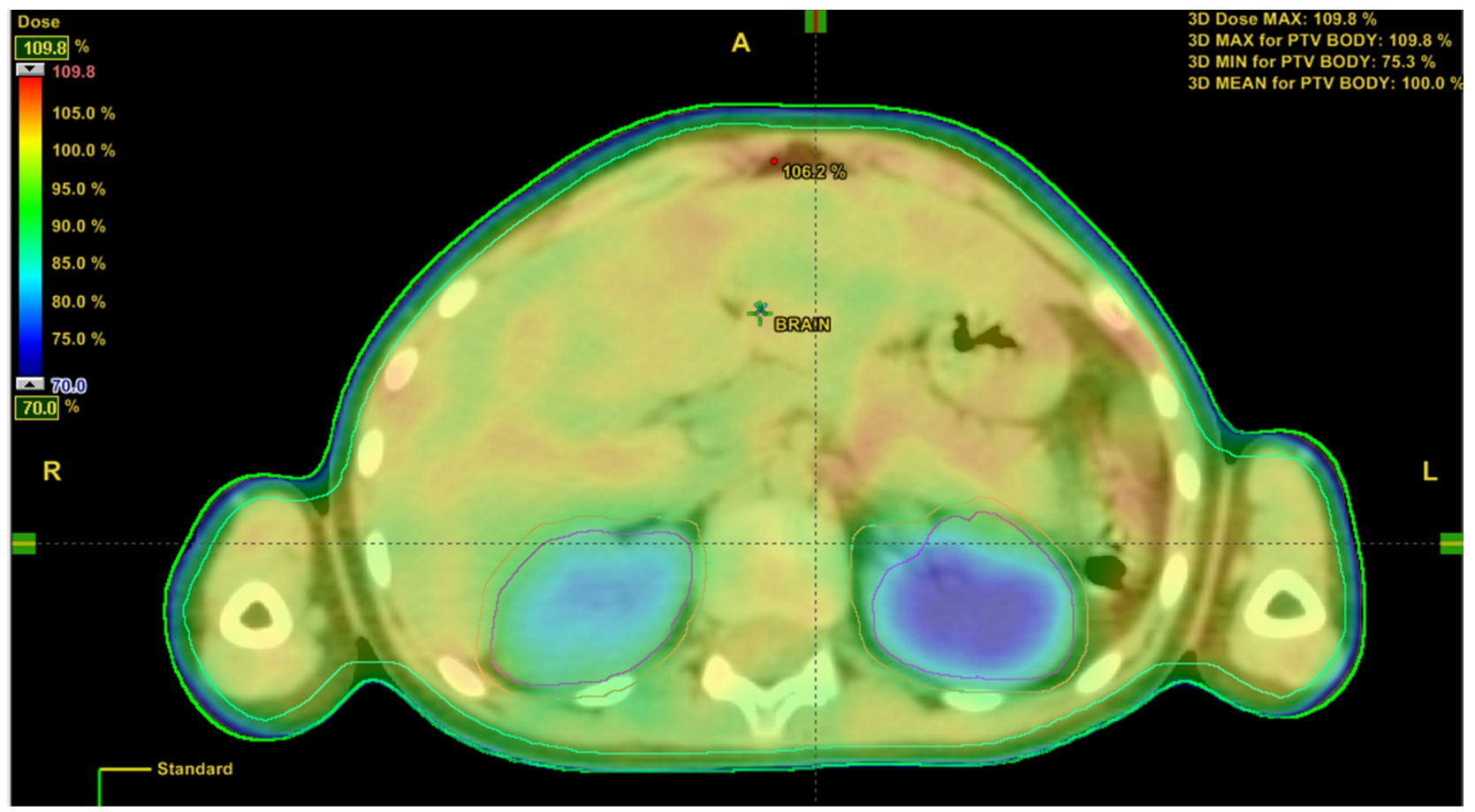

FIG. 2: RapidArc volumetric arc therapy TBI isodose distributions in color wash - Abdominal axial. Isodose lines shown correspond to: 8.4 Gy [70\% of prescribed dose].

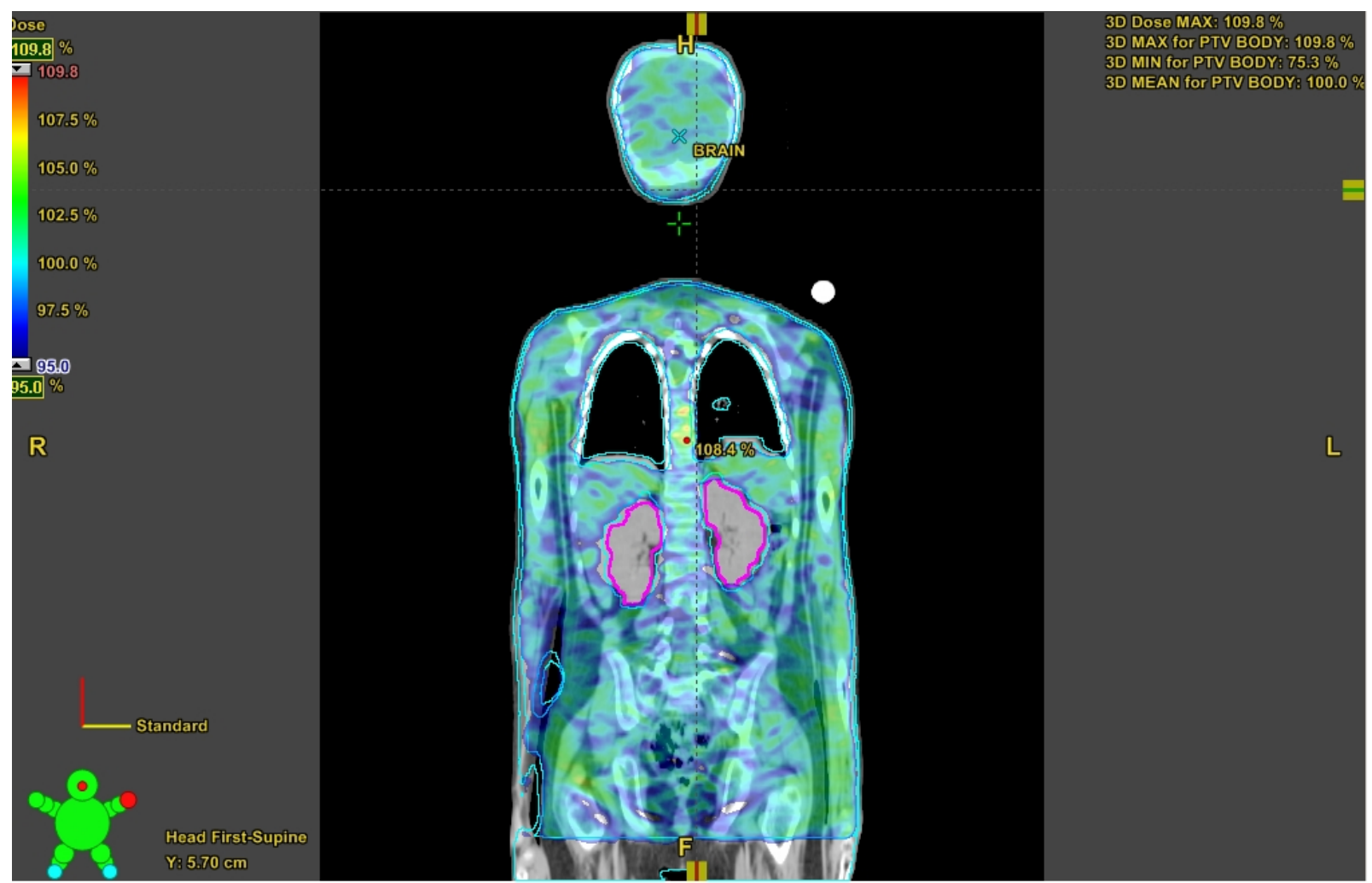

FIG. 3: Coronal section of body showing the isodose distribution in color wash. Isodose lines shown correspond to $11.4 \mathrm{~Gy}$ [95\% of the prescribed dose]. 


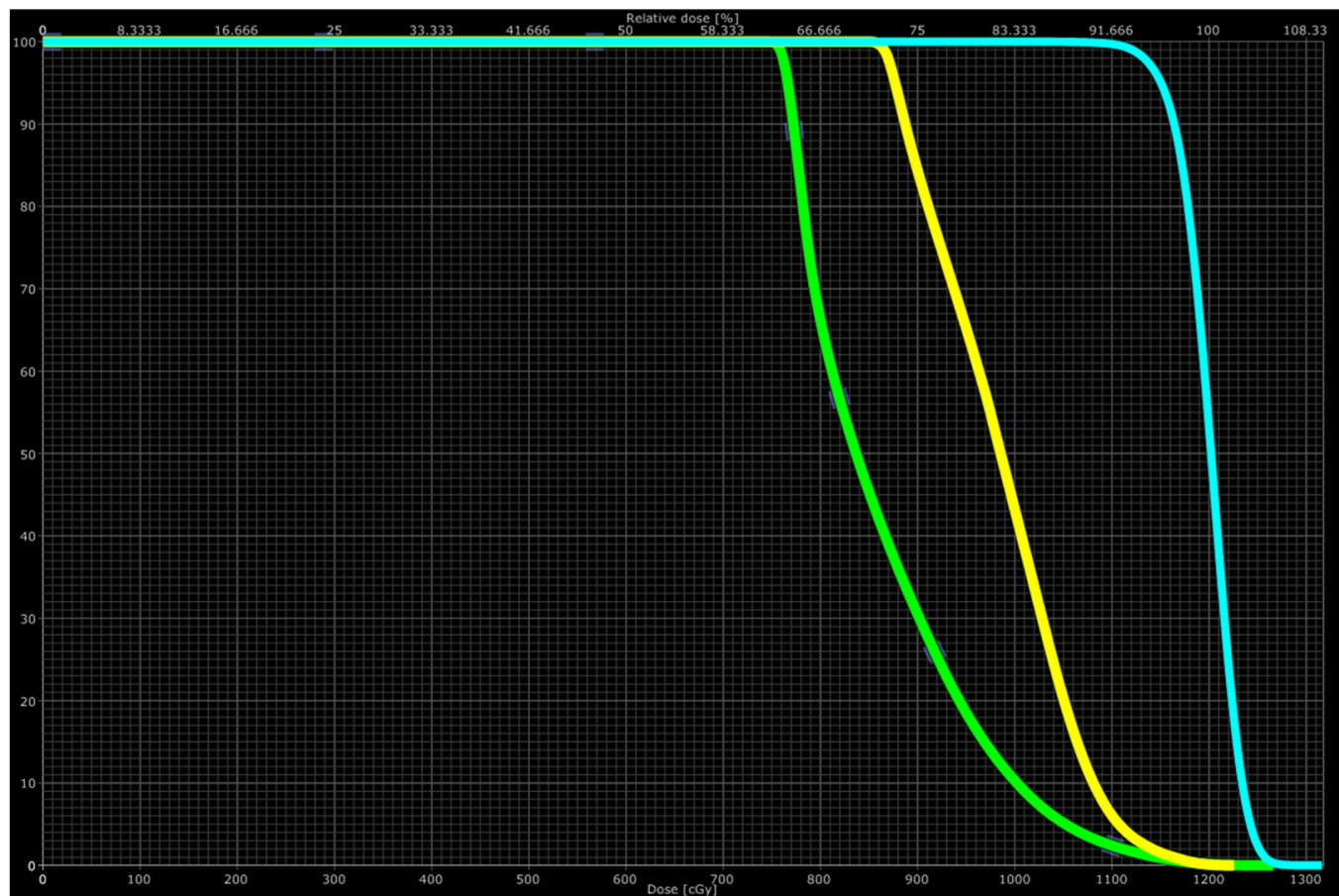

FIG. 4: Cumulative dose volume histogram. Planning target volume [PTV] in cyan color, kidney in yellow color and lung in green color.

\section{Discussion}

TBI is a valuable addition to hematopoietic stem cell transplantation programmes as it allows delivery of a uniform dose to entire body including all sanctuary sites regardless of blood supply. In addition chance of cross resistance with other systemic chemotherapeutic agents is less and there is no problem with altered dose intensity due to elimination through the excretory system. As per the ACR-ASTRO practice guidelines use of doses in the range of 12 - $15 \mathrm{~Gy}$ in 8 - 12 fractions over 4 days is ideal. ${ }^{14}$ In addition to a low dose per fraction and multiple treatments a low dose rate below 0.2 $\mathrm{Gy} / \mathrm{min}$ is associated with reduced risk of late side effects. Low dose TBI typically utilizes doses in the range of 2 - 8 Gy in 1- 4 fractions.

Unfortunately in a resource poor nation like India it is not possible to devote an entire machine for TBI given the low case load. Moreover implementation of TBI requires extensive quality assurance especially as several accessories and atypical patient positioning are involved. In addition to above time taken for each treatment extends well beyond 40 minutes for each session depending on the machine used for treatment. Hence most of the Indian institutes are reluctant to start TBI programs.

Recently attention has been focused on use of Rotational IMRT techniques like Helical Tomotherapy for TBI delivery. ${ }^{5,15}$ Delivering TBI using Helical Tomotherapy, however, requires the availability of the said machine which is one limiting factor in its widespread use. Another concern is that the nature of radiation delivery in Helical
Tomotherapy exposes only a small volume of the target to the radiation dose instantaneously. ${ }^{16}$ This is unlike conventional TBI where the entire blood pool and bone marrow gets irradiated simultaneously. Our present technique of treatment delivery using three isocentric arcs can minimize this issue as a larger volume of blood pool gets exposed to the radiation. Further the time taken to deliver each arc is of the order of 1 - 2 minutes which ensures that the entire blood pool gets exposed at each gantry rotation. Use of chemotherapy preconditioning regimens can further reduce the risk of missing circulating clonogenic cells.

Due to the unavailability of an anthropomorphic phantom we have used the Octavius Phantom for treatment verification. However, Aydogan et al. have used a similar Mapcheck phantom for absolute and relative dose verification of the treatment plan when delivering RapidArc for Total Marrow Irradiation. ${ }^{8}$ Chandraraj et al. have also shown that the PTW array along with a Octavius phantom yields equivalent dosimetric results on quality assurance of RapidArc IMRT as compared to EDR2 films. ${ }^{17}$ However we do plan to delivery and verify the plan using thermoluminescence dosimetry on an anthropomorphic phantom once it has been purchased.

A potential problem with TBI delivered using rotational techniques is that the instantaneous dose rate may not be less than 0.2 Gy per minute as recommended. ${ }^{14}$ In our present technique the average time taken to deliver two arcs at each isocenter was 2.48 minutes. Also the total dose delivered per fraction is 1.2 Gy per fraction and the total beam on time is 7.2 minutes the total dose rate comes to be $0.17 \mathrm{~Gy} / \mathrm{min}$. This compares favorably to the previously described Helical 
Tomotherapy technique where the instantaneous dose rates at each treated sub-volume are necessarily higher due to the nature of treatment delivery. A bigger advantage for resource constrained setting like ours is that this magnitude of total beam on time allows us to treat the patient on a standard treatment slot on the same machine with image guidance. In addition time and effort required for quality assurance is also less as the entire quality assurance can be performed within 20 minutes for a typical case and can be clubbed together with additional QA being performed on the given day.

The present planning strategy incorporated the creation of an optimization PTV which was trimmed $3 \mathrm{~mm}$ below the surface. It is to be noted that the average thickness of the skin is around $2-3 \mathrm{~mm}$. The present planning strategy has been extrapolated from areas requiring optimization for superficial tumors especially in the head neck region. In the present study, the TPS reported doses at the surface were 75 - $80 \%$. It is also known that the AAA algorithm can underestimate doses at $2 \mathrm{~mm}$ depth as compared to direct measurements by a mean of $4.71 \%(9.17 \%) .{ }^{18}$ We plan to conduct further studies of direct verification of superficial doses after acquisition of the anthropomorphic phantom using thermo-luminescence dosimetry for plans generated in multiple CT data-sets in the near future.

Use of RapidArc for delivering TBI was described in abstract form in a recent paper. ${ }^{19}$ However in this paper the patient was laid down in the floor and extended SSD partial 80 degree arcs were used. In contrast in our technique patient can rest in a supine position comfortably on the treatment couch itself and treatments can be conducted without requiring extended SSDs. Another advantage of the presently described technique is that it lends itself to image guidance very well with position verification using CBCT / $\mathrm{kV}$ imaging possible using on board imaging systems. Further additional beam data for extended SSD does not need to be generated saving precious physics time. Additional electron boosts for chest wall due to under-dosage arising from use of lung blocks as seen in conventional TBI treatments is also not required.

\section{Conclusion}

The study demonstrates that VMAT planning technique described here is feasible for TBI treatment. Unlike conventional TBI chest wall boost with electrons is not required and treatment can be done on the treatment couch without requiring extended SSD techniques. Further implementation and quality assurance of the process is simplified as compared to standing TBI with extended SSD. As patients can be treated in a supine position hence comfort and tolerability is likely to be significantly better also. The shorter time required would be beneficial to departments with limited machine resources.

\section{Conflict of interest}

The authors declare that they have no conflicts of interest. The authors alone are responsible for the content and writing of the paper.

\section{References}

1. Van Dyk J, Galvin JM, Glasgow GP, et al. The physical aspects of total and half body photon irradiation: a report of Task Group 29, Radiation Therapy Committee Association of Physicists in Medicine. American Association of Physicists in Medicine 1986.

2. Quast U. Whole body radiotherapy: A TBI-guideline. JMed Phys 2006; 31:5-12.

3. Miralbell R, Rouzaud M, Grob E, et al. Can a total body irradiation technique be fast and reproducible? Int J Radiat Oncol Biol Phys 1994; 29:1167-73.

4. Chen $\mathrm{HH}, \mathrm{Wu}$ J, Chuang KS, et al. Total body irradiation with step translation and dynamic field matching. Biomed Res Int 2013; 2013:216034.

5. Hui SK, Kapatoes J, Fowler J, et al. Feasibility study of helical tomotherapy for total body or total marrow irradiation. Med Phys 2005; 32:3214-24.

6. Zhuang AH, Liu A, Schultheiss TE, Wong JY. Dosimetric study and verification of total body irradiation using helical tomotherapy and its comparison to extended SSD technique. Med Dosim 2010; 35:243-9.

7. Teoh M, Clark CH, Wood K, et al. Volumetric modulated arc therapy: a review of current literature and clinical use in practice. Br J Radiol 2011; 84:967-96.

8. Aydogan B, Yeginer M, Kavak GO, et al. Total marrow irradiation with RapidArc volumetric arc therapy. Int J Radiat Oncol BiolPhys 2011; 81:592-9.

9. Mancosu P, Navarria P, Castagna L, et al. Anatomy driven optimization strategy for total marrow irradiation with a volumetric modulated arc therapy technique. J Appl Clin Med Phys 2012; 13:3653.

10. Surucu M, Yeginer M, Kavak GO, et al. Verification of dose distribution for volumetric modulated arc therapy total marrow irradiation in a humanlike phantom. Med Phys 2012; 39:281-8.

11. Han C, Schultheisss TE, Wong JYC. Dosimetric study of volumetric modulated arc therapy fields for total marrow irradiation. RadiotherOncol 2012; 102:315-20.

12. Zhuang $\mathrm{T}, \mathrm{Wu} \mathrm{Q}$. Generating arbitrary one-dimensional dose profiles using rotational therapy. Phys Med Biol 2010; 55:6263-77. 
13. Low DA, Harms WB, Mutic S, Purdy JA. A technique for the quantitative evaluation of dose distributions. Med Phys 1998; 25:656-61.

14. Wolden SL, Rabinovitch RA, Bittner NHJ, et al. American College of Radiology (ACR) and American Society for Radiation Oncology (ASTRO) practice guideline for the performance of total body irradiation (TBI). Am J Clin Oncol 2013; 36:97-101.

15. Peñagarícano JA, Chao M, Van Rhee F, et al. Clinical feasibility of TBI with helical tomotherapy. Bone Marrow Transplant 2011; 46:929-35.

16. Schultheiss TE, Wong J, Liu A, et al. Image-guided total marrow and total lymphatic irradiation using helical tomotherapy. Int J Radiat Oncol Biol Phys 2007; 67:1259-67.

17. Chandraraj V, Stathakis S, Manickam R, et al. Comparison of four commercial devices for RapidArc and sliding window IMRT QA. J Appl Clin Med Phys 2011; 12:3367.

18. Oinam AS, Singh L. Verification of IMRT dose calculations using AAA and $\mathrm{PBC}$ algorithms in dose buildup regions. J Appl Clin Med Phys 2010; 11:3351.

19. Evans MDC, Ruo R, Seuntjens J, et al. Poster-Thur Eve-31: RapidArc total body photon irradiation: A feasibility study. Med Phys 2012; 39:4630. 